\title{
1 Azacytidine targeting SARS-CoV-2 viral RNA as a potential treatment for
}

\section{COVID-19}

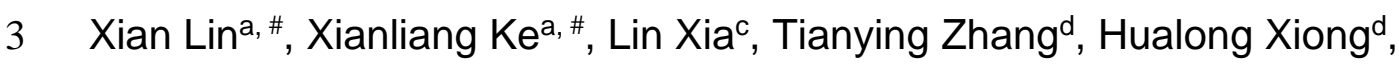

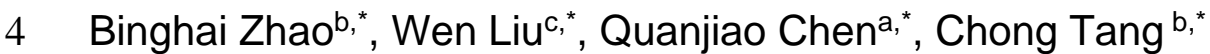

5

6 aCAS Key Laboratory of Special Pathogens and Biosafety, Wuhan Institute of

7 Virology, Center for Biosafety Mega-Science, CASCIRE, Chinese Academy of

8 Sciences, 430071, Wuhan, China.

9 bDepartment of Pathophysiology, School of Medicine, Beihua University, Jilin

10 132011, Jilin, China;

11 'Fujian Provincial Key Laboratory of Innovative Drug Target Research, School

12 of Pharmaceutical Sciences, Xiamen University, Xiamen, Fujian 361102,

13 China;

14 dState Key Laboratory of Molecular Vaccinology and Molecular Diagnostics,

15 National Institute of Diagnostics and Vaccine Development in Infectious

16 Diseases, School of Public Health, Xiamen University, Xiamen, Fujian 361102 ,

17 China;

18 * Correspondence: Binghai Zhao, email:zhaobinghai01@vip.163.com; Wen

19 Liu, email: w2liu@xmu.edu.cn; Quanjiao Chen (chenqj@wh.iov.cn); Chong

20 Tang, leochong718@gmail.com

21 \#These authors contributed equally to this work. 
23

24

\section{Abstract}

The COVID-19 pandemic is a global health disaster. Moreover, emerging mutated virus strains present an even greater challenge for existing vaccines and medications. One possible solution is to design drugs based on the properties of virus epigenome, which are more common among coronaviruses. Here, we reported an FDA-approved drug for myelodysplastic syndrome, azacytidine (5Aza), limited virus infection and protected mice against SARS-CoV-2. We demonstrated that this antiviral effect is related to $5 \mathrm{Aza}$ incorporation into viral RNA, which disrupt m5C RNA methylation modification profile. This work suggests that targeting viral epigenomes is a viable therapeutic strategy, potentially opening new pathways for treating COVID-19. 

and medications. One possible solution is to design drugs based on the properties of virus epigenome, which are more common among coronaviruses. structural analog of cytidine (Fig.S1). It exhibits antiviral effects against several viruses. However, it is unknown whether this extends to SARS-CoV-2. cells, with half-maximal inhibitory concentration $\left(\mathrm{IC}_{50}\right)=6.99 \mu \mathrm{M}$ and selective index $(\mathrm{SI})=20.41$ (Fig.1a). The viral titer in cell supernatants detection (Fig.1b) and indirect immunofluorescence assay (IFA) against viral $\mathrm{N}$ protein (Fig.1c) revealed 5Aza restricted SARS-CoV-2 infection in a dose-dependent manner. Time-of-drug-addition assay (Fig.S2a) showed that 5Aza functioned after virus entry (Fig.S2b, c, and d). The virus replication is still $50 \%$ suppressed when $5 \mathrm{Aza}$ at $32 \mu \mathrm{M}$ is added 24 hours after infection (Fig. S3). with a mouse-adapted SARS-CoV-2 (MA-SARS-CoV-2) that exhibits high infectivity in mice. In clinical use, the recommended starting dose of $5 \mathrm{Aza}$ is 75 $\mathrm{mg} / \mathrm{m}^{2}$ (equals to $\sim 2 \mathrm{mg} / \mathrm{kg}$ ) in treating MDS. Therefore, mice were intranasally challenged with $2 \times 10^{3}$ plaque-forming units (PFU) of MA-SARS-CoV-2, 
69 (equal to $6 \mathrm{mg} / \mathrm{m}^{2}$ surface area) at $1 \mathrm{~d}$ post infection (dpi), once daily for seven

70 consecutive doses. As shown in Fig.1d, at 5 dpi, mice in 5Aza-treated group

71 started to recovery body weight, while mice body weight in saline-treated group

72 were still decreasing and $5 / 6$ of mice lost more than $25 \%$ of body weight.

73 In accordance with this, $83.33 \% \quad(5 / 6)$ of saline-treated animals became

74 moribund (defined as $25 \%$ loss of body mass) compared with $16.67 \%(1 / 6)$ in

75 5Aza-treated group. Besides, the viral RNA copy number and titers in the

76 lungs of 5Aza-treated mice also showed a significant decrease (Fig.1f).

77 Moreover, histological examination revealed remarkable amelioration of lung

78 damage at $4 \mathrm{dpi}$ in the 5Aza group (Fig.S4c). In contrast, the saline group

79 exhibited massive alveolar space mononuclear cell infiltration, moderately

80 severe bronchiolar epithelial cell death, and intra-endothelium and

81 perivascular infiltration of pulmonary blood vessels (Fig.S4b). The RNA-seq of

82 lungs also demonstrated that the 5Aza rescued most downregulated genes

83 with virus infection (Fig.S5), further demonstrated that $2 \mathrm{mg} / \mathrm{kg} 5 \mathrm{Aza}$ treatment

84 protected against SARS-CoV-2 attack. Based on the dose used in mice is

85 equivalent to that in treating MDS in humans, it would be encouraging to

86 extend to human COVID therapy.

As an RNA analog with $\mathrm{OH}-g$ roup on ribose $2^{\prime}$ carbon (2' C) (Fig.S1a),

88 5Aza could theoretically incorporate into RNAs. High-resolution mass

89 spectrometry analysis showed that RNA containing 5Aza increased $\sim 40$-fold 
90 with short-term 5Aza treatment (Fig.S6). We took advantage of 5Aza stability

91 in bisulfite treatment to develop a new method (5Aza-BSseq) that identifies the

92 location where 5Aza is incorporated (Fig.S7). Notably, 5Aza-BSseq results

93 also suggested efficient azacytidine incorporation into viral RNA (Fig.1g).

94 After deoxidized conversion to decitabine and incorporation into DNA, 5Aza

95 causes endogenous retrovirus (ERV) DNA hypomethylation, which activates

96 retroviral RNA transcription and triggers type I interferon response. In this study,

97 decitabine was five times less efficient than 5Aza in inhibiting viral replication

98 (Fig.S8). Additionally, 5Aza did not significantly increase ERV expression

99 (Fig.S9). Therefore, 5Aza incorporation into RNA might be linked to its

100 inhibitory effects on viral replication.

101 We further explored the possible antiviral mechanism of 5Aza

102 incorporation into viral RNA. Previous research showed that $5 \mathrm{Aza}$

103 incorporation enhanced lethal mutagenesis on influenza virus. However, we

104 found consistent mutations and comparable mutation rates in the viral RNA

105 propagated in 5Aza-treated or control cells (Supplementary Table 1),

106 excluding the role of 5Aza-induced lethal mutagenesis in anti-SARS-CoV-2.

107 Considering that 5Aza sequesters tRNA methyltransferase to inhibit

108 cytosine-C5 methylation $\left(\mathrm{m}^{5} \mathrm{C}\right)$ in $\mathrm{tRNA}^{1}$ (the reaction principle is exhibited in

109 Fig.S10), we further explored whether 5Aza incorporation affects viral RNA

110 methylation. We used nanopore direct RNA sequencing to avoid false-positive 
111 methylation sites caused by unconverted $5 \mathrm{Aza}$, as assessed with bisulfite

112 sequencing. The nanopore $\mathrm{m}^{5} \mathrm{C}$ identification algorithm, trained by $\mathrm{m}^{5} \mathrm{C}$ control

113 data as well as many datasets, was used for data analysis. High-confidence

$114 \mathrm{~m}^{5} \mathrm{C}$ sites in 5 Aza-treated viral RNA decreased significantly by $40 \%$ (false

115 positive < 0.05) (Fig.1h). We further validated these $\mathrm{m}^{5} \mathrm{C}$ sites using the

116 optimized RNA-BSseq and strict criteria (Fig.S11 and $\mathrm{S} 12$ ). All $\mathrm{m}^{5} \mathrm{C}$ sites

117 identified through nanopore sequencing were also present in RNA-BSseq

118 (Fig.S13).

119 The primary writers for $\mathrm{m}^{5} \mathrm{C}$ on mRNAs have been proposed to be NSUN2

120 and DNMT2, which are demonstrated to contribute to m5C methylation on

121 HIV-1 RNA and thus facilitate virus infection ${ }^{2,3}$. Here, we found that

122 overexpression of DNMT2 and NSUN2, significantly promoted the

123 SARS-CoV-2 replication (Fig.S14), implying both of DNMT2 and NSUN2 are

124 responsible for the $\mathrm{m}^{5} \mathrm{C}$ methylation of SARS-CoV-2 RNA. The

125 immunoprecipitation (IP) assay showed that DNMT2 and NSUN2 could bind to

126 SARS-CoV-2 RNA (Fig. 1i). Notably, DNMT2 IPed more RNAs in the presence

127 of 5Aza. As RNA methylation occurs, cytosine RNA methyltransferases

128 ( $\mathrm{m}^{5} \mathrm{C}-\mathrm{RMTs}$ ) form a covalent thioester bond, connecting the cysteine residue

129 of its catalytic domain to the $\mathrm{C} 6$ position, thereby forming an $\mathrm{m}^{5} \mathrm{C}-\mathrm{RMT}-\mathrm{RNA}$

130 adduct. Next, RMT transfers a methyl group from the cofactor S-adenosyl

131 methionine (SAM) to C5 of cytosine, followed by enzyme release from the 
132 adduct $\beta$-elimination. $5 \mathrm{Aza}$ is a suicide mechanism-based inhibitor of

$133 \mathrm{~m}^{5} \mathrm{C}-\mathrm{RMTs} \mathrm{s}^{4}$. RNAs containing $5 \mathrm{Aza}$ at the precise target site will sequester the

$134 \mathrm{~m}^{5} \mathrm{C}-\mathrm{RMT}$ by generating RNA-m ${ }^{5} \mathrm{C}-\mathrm{RMT}$ adducts, which will result in the

135 decreased level of active endogenous enzymes ${ }^{5}$. Consistent with this theory,

136 we observed an obvious decreased DNMT2, rather than NSUN2 protein upon

137 5Aza treatment in SARS-CoV-2 infected cells (Fig. S15). It suggests that

138 DNMT2 is more likely the main enzyme for $m^{5} \mathrm{C}$ RNA methylation in

139 SARS-CoV-2. Further investigations are underway to elucidate the relevance

140 of $\mathrm{m}^{5} \mathrm{C}$ methylation and SARS-CoV-2 infection to 5Aza treatment.

141 In summary, we demonstrated that 5Aza can incorporate into

142 SARS-CoV-2 RNA and disturb $\mathrm{m}^{5} \mathrm{C}$ RNA methylation modification, potentially

143 contributing to 5Aza's anti-SARS-CoV-2 activity. We repurposed 5Aza as a

144 promising candidate for combating COVID-19 and introduced the possibility of

145 targeting viral epigenomes as a novel antiviral strategy.

148 References

1491 Khoddami, V. \& Cairns, B. R. Identification of direct targets and modified

150 bases of RNA cytosine methyltransferases. Nature Biotechnology 31, 458-464,

151 doi:10.1038/nbt.2566 (2013).

1522 Dev, R. R. et al. Cytosine methylation by DNMT2 facilitates stability and

153 survival of HIV-1 RNA in the host cell during infection. Biochem J 474,

154 2009-2026, doi:10.1042/BCJ20170258 (2017). 
1553 Courtney, D. G. et al. Epitranscriptomic Addition of m(5)C to HIV-1

156 Transcripts Regulates Viral Gene Expression. Cell Host Microbe 26, 217-227

157 e216, doi:10.1016/j.chom.2019.07.005 (2019).

1584 Santi, D. V., Garrett, C. E. \& Barr, P. J. On the mechanism of inhibition of

159

160

161

162

163

164

165

166

167

Major Projects (grant number 2020ZX10001016).

174

175

The authors declare no competing interests.

177

178

Fig. 1 Azacytidine targets SARS-CoV-2 RNA to inhibit virus infection. DNA-cytosine methyltransferases by cytosine analogs. Cell 33, 9-10, doi:10.1016/0092-8674(83)90327-6 (1983).

5 Schaefer, M., Hagemann, S., Hanna, K. \& Lyko, F. Azacytidine inhibits RNA methylation at DNMT2 target sites in human cancer cell lines. Cancer Res 69, 8127-8132, doi:10.1158/0008-5472.CAN-09-0458 (2009).

\section{Acknowledgements}

We thank Tao Du, Jin Xiong, and other colleagues from BSL-3 Laboratory of

Wuhan Institute of Virology and Wuhan National Biosafety Laboratory for their critical support and excellent coordination. We thank Prof. Meilin Jin of Huazhong Agricultural University for kindly providing mouse-adaptived SARS-CoV-2 strain. We thank Editage (www.editage.cn) for English language editing. This work was supported by the National Science and Technology

\section{Competing interest}

\section{Figure legend}

a, b, and $\mathbf{c}$ The anti-SARS-CoV-2 effect of Azacytidine in vitro. Vero E6 cells 
182 doses of Azacytidine. The chloroquine was used as a drug control. At 24 hpi,

183 cell supernatants were collected. a IC50 and EC 50 were calculated by detecting

184 viral RNA through RT-PCR and CCK-8 assay, respectively. Left and right

185 Y-axes represent mean \% inhibition of virus and \% cytotoxicity of $5 \mathrm{Aza}$,

186 respectively. b Cell supernatants were collected at $24 \mathrm{hpi}$, and the viral titers

187 were measured using the plaque assay. c Immnofluorescence microscopy of

188 virus infection via probing $\mathrm{N}$ protein of SARS-CoV-2. Bars, $200 \mu \mathrm{M}$. d, e, and $\mathbf{f}$

189 The anti-SARS-CoV-2 effect of Azacytidine in vivo. 6-7 weeks old female

190 BALB/c mice were randomly divided into three groups with 9 mice per group.

191 Mice were intranasally challenged with $2 \times 10^{3}$ PFU MA-SARS-CoV-2 in $50 \mu \mathrm{l}$

192 DMEM (infection groups) or equal DMEM (mock infection). At 1 dpi, mice were

193 intraperitoneally injected with $2 \mathrm{mg} / \mathrm{kg}$ 5Aza (SARS-CoV-2+5Aza group) or an

194 equivalent volume of sterile saline (SARS-CoV-2+saline and mock infection

195 groups), once daily for seven consecutive doses. $\mathbf{d}$ body weight was measured

196 daily for $7 \mathrm{~d}(\mathrm{n}=6)$, mice with $25 \%$ body weight loss were considered moribund

197 and euthanized. Note: the remaining one mouse (body weight loss less than

198 25\%) in SARS-CoV-2+saline group was not recorded at dpi 6 and 7. e the

199 survival rates of mice $(n=6)$; mice with more than $25 \%$ of body weight loss in d

200 were considered moribund and euthanized. $\mathbf{f}$ at $4 \mathrm{dpi}$, three mice per group

201 were euthanized for detecting viral RNA copy and virus titer in the lungs. $\mathbf{g}$ The

202 pseudo $\mathrm{m}^{5} \mathrm{C}$ locations indicated incorporated azacytidine. Vero-E6 cells were 
203 infected with 1 moi SARS-CoV-2 in the presence of $10 \mu \mathrm{M} 5 \mathrm{Aza}$ or saline.

204 After $12 \mathrm{~h}$, total RNA was isolated and comparison of 5Aza-treated viral RNA

205 with the control using bisulfite sequencing was performed; the non-overlapping

206 points are pseudo $\mathrm{m}^{5} \mathrm{C}$ locations that indicate where $5 \mathrm{Aza}$ was incorporated. $\mathbf{h}$

207 RNAs of cells infected with SARS-CoV-2 that treated with or without 5Aza

208 were subjected to Nanopore direct RNA sequencing. i Vero E6 cells transiently

209 overexpressing DNMT2, NSUN2, or GFP with a HA tag were infected with 0.2

210 moi SARS-CoV-2 for $20 \mathrm{~h}$, in the presence of $16 \mu \mathrm{M} 5 \mathrm{Aza}$ or not. Lysates were

211 prepared and split for incubation with mouse anti-HA antibody. Co-precipitated

212 RNA was analyzed by qRT-PCR using primer sets targeting viral S gene. The

213 level of viral RNA amplicon was determined as the percentage of input ( $1 \%$ of

214 lysate) (right); the expression of indicated protein and products of IP was

215 validated by western blot (left). Data are means \pm SD analyzed using Student's

$216 t$ test or One-way ANOVA (body weight change); Log-rank test was used to

217 analyze the significance of survival differences. ${ }^{*} p<0.05,{ }^{* *} p<0.01,{ }^{* *} p<$

$218 \quad 0.001$. 
bioRxiv preprint doi: https://doi.org/10.1101/2021.09.01.458475; this version posted September 2, 2021. The copyright holder for this preprint (which was not certified by peer review) is the author/funder, who has granted bioRxiv a license to display the preprint in perpetuity. It is made available under aCC-BY-NC-ND 4.0 International license.

\section{Fig. 1}
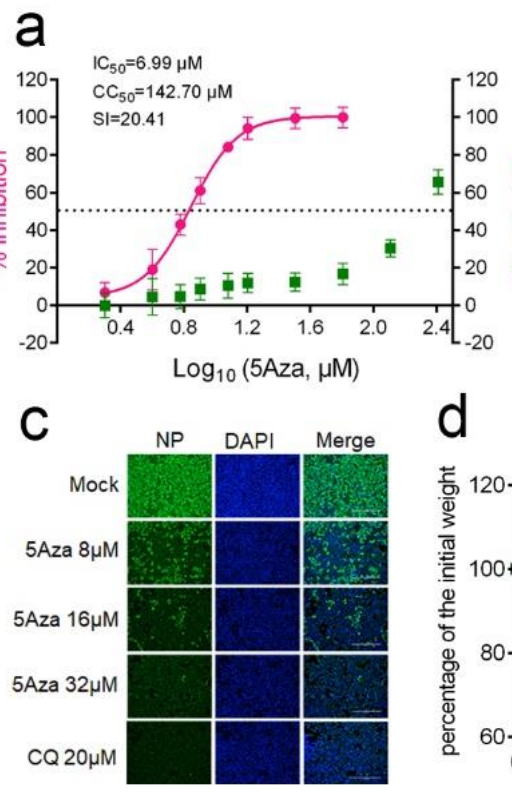

f

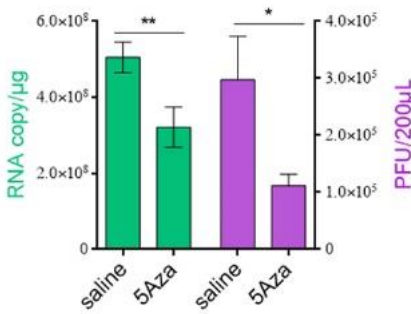

i

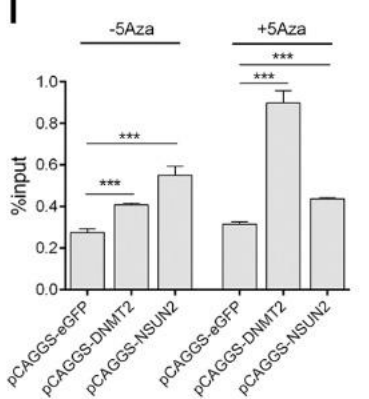

g b
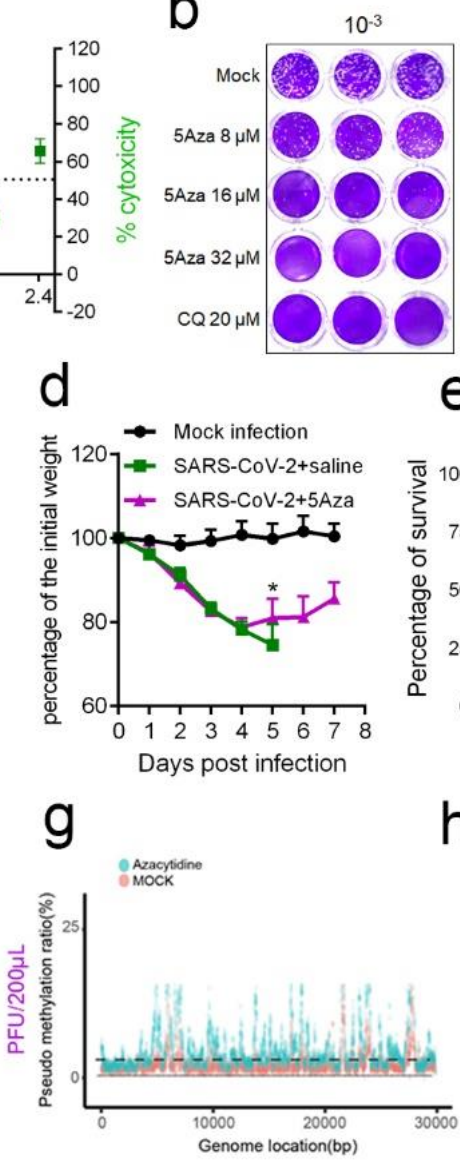

e Mock infection

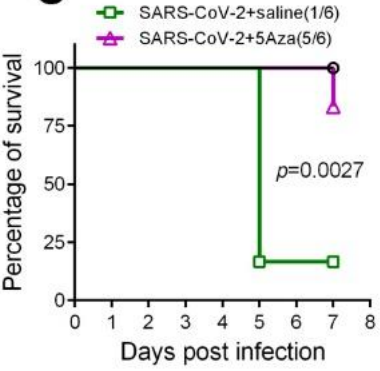

$\mathrm{h}$

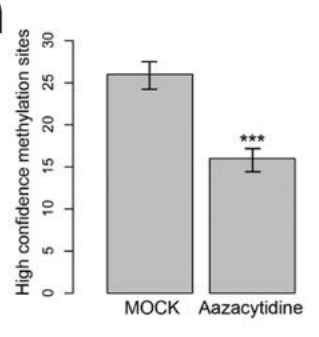

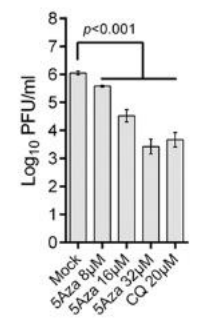

saline(1/6) $\approx$ SARS-CoV-2+5Aza(5/6)

Days post infection

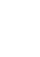

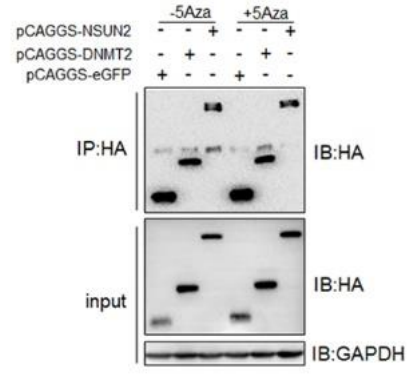

